\title{
Methylprednisolone therapy in multiple sclerosis: a profile of adverse effects
}

\author{
P R LYONS, P K NEWMAN, M SAUNDERS \\ From the Department of Neurology, Middlesbrough General Hospital, Cleveland, UK
}

SUMMARY A retrospective analysis of 350 treatment courses using high dose pulsed intravenous methylprednisolone for relapses of multiple sclerosis revealed a low number of adverse effects. This study confirms that high dose methylprednisolone is a safe therapeutic option in multiple sclerosis.

A small number of controlled clinical trials comparing short courses of prednisolone ${ }^{1}$ or $\mathrm{ACTH}^{2}$ with a placebo have shown a limited beneficial effect in acute relapse of multiple sclerosis. Recently the effect of higher doses of steroid in shorter courses has been investigated and these studies have shown a favourable response when high dose pulsed intravenous methylprednisolone treatment is compared with $\mathrm{ACTH}^{3}$ or with placebo. ${ }^{4}$ Several uncontrolled studies using high dose methylprednisolone ${ }^{5-7}$ have supported these findings, and all suggest that in addition to the beneficial aspects, such treatment is safe, well tolerated and with few adverse effects. The present study looks at a population of patients with multiple sclerosis treated with intravenous methylprednisolone with particular emphasis on the nature and frequency of unwanted effects.

\section{Patients and methods}

A retrospective review of case records gave a total of 240 patients having definite or probable multiple sclerosis treated between mid 1978 and 1987 with intravenous methylprednisolone pulse therapy. In nearly all cases there had been a worsening of symptoms in the weeks prior to the treatment, but in a few the intervention was an attempt to influence a chronic progressive course. One hundred and sixty four patients received a single course of treatment, 54 received two courses, 15 received three, five received four

Address for reprint requests: $\operatorname{Dr} \mathbf{P} \mathbf{R}$ Lyons, Department of Neurology, Middlesbrough General Hospital, Middlesbrough, Cleveland, T55 5A2, UK.

Received 14 April 1987. Accepted 8 September 1987 and there were two patients who received six and seven courses respectively, giving a total of 350 drug administrations. The average age at the time of treatment was 34 years with a range from 18 to 57 years. The standard treatment regime consisted of $1 \mathrm{~g}$ of intravenous methylprednisolone infused over 20 to 30 minutes, and administered daily for 5 days followed by oral prednisolone tailing from $60 \mathrm{mg}$ daily to zero over 10 days. The largest dose given to a single individual was a total of $30 \mathrm{~g}$ of methylprednisolone in six treatment episodes over 5 years. The patients were monitored before and during the treatment course and were usually reviewed at 1 to 3 months and then followed on a regular basis.

\section{Adverse reactions}

(1) Minor infections Two female patients required treatment for lower urinary tract infections which became symptomatic whilst receiving steroids and two other patients developed oral and vaginal candidiasis respectively which required the use of antifungal agents. There were no cases of disseminated infection or septicaemia.

(2) Epilepsy In one patient a single seizure occurred within 12 hours of the end of a course of treatment. Two other patients later developed epilepsy, one suffering a first seizure during a light general anaesthetic 24 days post-steroid treatment and a second patient had a first seizure 4 years after treatment. In 25 patients a standard 16 channel electroencephalogram was obtained pre- and immediately posttreatment and there was no significant difference between the two sets of records.

(3) Hyperglycaemia All patients had a random or fasting blood glucose estimation prior to administration of methylprednisolone and underwent daily analysis for glycosuria. These routine examinations revealed an elevated pretreatment random blood glucose in one obese female who had an uneventful steroid course, but 1 year later required insulin for proven diabetes. Another $11(4 \cdot 6 \%)$ patients were 
noted to have glycosuria and an elevated blood glucose during treatment with methylprednisolone but they did not subsequently require intervention.

(4) Gastrointestinal symptoms Upper abdominal dyspeptic pain was recorded in five cases, and treated with an antacid and/or an $\mathrm{H} 2$ antagonist, but only in one case was the course of methylprednisolone suspended. In one patient symptoms persisted and at endoscopy 4 weeks after treatment a severe duodenitis was demonstrated which required a prolonged course of an $\mathrm{H} 2$ antagonist. There were several reports of nausea which did not need any specific treatment.

(5) Psychiatric symptoms A feeling of well being was common but there were four recorded episodes of euphoria and two reports of depression. One patient with a pre-treatment organic mental disturbance experienced the onset of psychotic depression during the administration of methylprednisolone.

(6) Minor adverse effects Minor side effects, including transient facial flushing, a brief disturbance of taste, distal paraesthesia, insomnia and mild weight gain, occurred in a significant proportion of patients but these were often not recorded. A survey of this kind is liable to underestimate the frequency of such minor effects.

(7) Other adverse effects In three patients an exacerbation of acne occurred. Ankle oedema was recorded in two, and one of these had diuretic treatment for 3 days. During treatment one patient was found to be hypertensive, but this resolved without intervention. In one instance a heel blister progressed to a small bed sore following treatment, and in another patient a localised area of subcutaneous necrosis followed extravasation of part of the injection. Ten days following completion of treatment a deep vein thrombosis developed in one patient. A steroid "let down" was seen in a small group of patients but no Addison crises followed the abrupt withdrawal of the high dose steroid regime. During the period of follow up there have been no steroid induced cataracts and no avascular necrosis of bone.

\section{Discussion and conclusions}

Pulsed intravenous methylprednisolone is widely used in the treatment of immune mediated disorders and substantial experience has accrued in organ transplantation, rheumatoid arthritis and in other connective tissue diseases. In most cases it has been a safe treatment ${ }^{8-10}$ but cautionary reports of serious side effects have appeared; these include life threatening infections $^{11}$ and sudden death probably due to cardiac arrhythmia and circulatory collapse. ${ }^{12}{ }^{13}$ However, there has been a background of general debility in these patients who are therefore very different from the generally fit and comparatively young group of multiple sclerosis sufferers. The present study indicates the overall safety of pulsed methylprednisolone in the treatment of multiple sclerosis.

In non-neurological disease an association between high dose methylprednisolone and seizures has been observed in a number of instances ${ }^{121415}$ and it has been suggested that alteration in brain water content induced by steroid treatment may be responsible. ${ }^{16}$ In one patient with multiple sclerosis a seizure appeared directly related to methylprednisolone treatment. However, it must be remembered the epilepsy occurs in approximately $2 \%$ of patients with multiple sclerosis $^{17}$ and the EEG is abnormal in up to $50 \%{ }^{18}$

Gastrointestinal complaints were infrequent and only in one case was endoscopy and long term $\mathrm{H} 2$ antagonist treatment required. This low frequency accords with other studies which have looked at the use of steroids ${ }^{19}$ and indicates that while caution is advisable where peptic symptoms are present, the use of methylprednisolone is unlikely to cause severe upper gastrointestinal upset. Hyperglycaemia was not encountered as a major problem and psychiatric complications were also minor in all but one case.

Our selection of patients for treatment with pulsed methylprednisolone remains broadbased, the only absolute contraindications being concurrent sepsis, organic mental disturbance or psychosis, active peptic ulceration and previous adverse reaction to methylprednisolone. It has been our practice to screen all patients for hyperglycaemia, but its presence does not preclude treatment. Multiple treatments have not led to complications but only rarely have courses of methylprednisolone been given at less than 6 monthly intervals.

A small study involving 20 patients and examining the effect of one pulse of methylprednisolone compared with five has been performed in this department. Although the results are not statistically significant they do suggest that the shorter course may be inadequate, as several patients who derived? little benefit from one pulse, subsequently improved their functional and disability scores when a full five pulse course of treatment was given. Hence it is our continuing practice to administer five pulses of methylprednisolone but in view of the efficacy of smaller dosages observed by other workers ${ }^{4}$ each pulse has been reduced to $500 \mathrm{mg}$.

This study may have underestimated minor symptoms, but more significant adverse reactions have been faithfully recorded and yet have appeared only infrequently. Our experience with 350 treatment courses of high dose pulsed intravenous methylprednisolone confirms that when used judiciously this is a safe therapeutic option in multiple sclerosis.

\section{References}

1 Eadie MJ, Tyrer JH. Neurological Clinical Pharmacology. Lancaster: MTP Press, International Medical Publishers, 1980.

2 Rose AS, Kuzma JW, Kurtzke JF, et al. Cooperative study in the evaluation of therapy in multiple sclerosis: ACTH vs placebo. Final report. Neurology 1970;20:1-59.

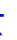


3 Barnes MP, Bateman DE, Cleland PG, et al. Intravenous methylprednisolone for multiple sclerosis in relapse. J Neurol Neurosurg Psychiatry 1985;48:157-9.

4 Milligan NM, Newcombe R, Compston DAS. A doubleblind controlled trial of high dose methylprednisolone in patients with multiple sclerosis: 1 . clinical effects. J Neurol Neurosurg Psychiatry 1987;50:511-6.

5 Dowling PC, Bosch UV, Cook SK. Possible beneficial effect of high dose intravenous steroid therapy in acute demyelinating disease and transverse myelitis. Neurology 1980;30:33-6.

6 Buckley C, Kennard C, Sawsh M. Treatment of acute exacerbations of multiple sclerosis with intravenous methylprednisolone. J Neurol Neurosurg Psychiatry 1982;45:179-80.

7 Newman PK, Saunders M, Tilley PJB. Methylprednisolone therapy in multiple sclerosis. $J$ Neurol Neurosurg Psychiatry 1982;45:941-2.

8 Cathcart ES, Idelson BA, Sheinberg MA, Couser WG. Beneficial effects of methylprednisolone "pulse" therapy in proliferative lupus nephritis. Lancet 1976;i:163-6.

9 Neild GH, Lee HA. Methylprednisolone pulse therapy in the treatment of polyarteritis nodosa. Postgrad Med 1977;53:382-7.

10 Williams IA, Baylis EM, Shipley ME. A double-blind placebo-controlled trial of methylprednisolone pulse therapy in active rheumatoid disease. Lancet 1982;ii:237-40.

11 Garrett R, Paulus H. Complications of intravenous methylprednisolone pulse therapy (abstract). Arthritis Rheum 1980;23:677.

12 Stubbs SS, Morrell RM. Intravenous methylprednisolone sodium succinate: adverse reactions reported in association with immunosuppressive therapy. Transplant Proc 1973;5:1145-6.

13 Moses RE, McCormick A, Nickey W. Fatal arrhythmia after pulsed methylprednisolone therapy. Ann Intern Med 1981;95:781-2.

14 Suchman AL, Condemi JJ, Leddy JP. Seizure after pulse therapy with methylprednisolone. Arthritis Rheum 1983;26:117.

15 Durrant S, Chipping PM, Palmer S, Garden-Smith EC. Cyclosporin, Methylprednisolone and convulsion. Lancet 1982;ii:829-30.

16 Cerrilli J, Miller JA. The effect of massive pulse steroid therapy on water content of the rat brain. Transplantation 1972;14:403-5.

17 Matthews WB. Epilepsy and disseminated sclerosis. $Q J$ Med 1962;31:141-55.

18 Funkhouse JB, Nagler B. The electroencephalogram in multiple sclerosis. Dis Nerv Syst 1959;20:41-3.

19 Do corticosteroids cause peptic ulcers? Drug Ther Bull 1987;25:41-2. 